\title{
Current ERCP practice in Belgium: the BSGIE survey
} On behalf of the board of the Belgian Society of Gastrointestinal Endoscopy (BSGIE) : C. Snauwaert ${ }^{1}$, X. Dekoninck $^{2}$,
T. Moreels

(1) Department of Hepatology and Gastroenterology, AZ Sint-Jan Brugge-Oostende AV, Ruddershove 10, 8000 Bruges ; (2) Department of Hepatology and Gastroenterology, Clinique Saint Pierre, Ottignies ; (3) Department of Hepatology and Gastroenterology, Cliniques Universitaires Saint-Luc, Brussels.

\begin{abstract}
Background and study aims: Data on procedural outcome and quality of endoscopic retrograde cholangiopancreatography (ERCP) in Belgian practice are scarce. The aim of this study is to assess current status of ERCP-performance in Belgium.

Methods : National multi-institutional survey (online questionnaire) among members of the Belgian Society of Gastrointestinal Endoscopy (BSGIE), conducted in the period June-August 2018. The RIZIV/INAMI provided real-life data on the total number of ERCPs performed in Belgium.

Results: Forty-five responders completed the survey (for 43 centers performing ERCP), providing information for 8368 ERCPs performed in $45 \%(43 / 95)$ of institutions performing ERCP in Belgium. Fifty-eight percent of centers performed $>100$ ERCPs/year and $7 \%$ of centers $(n=3)$ performed $<50$ ERCPs/year. According to the RIZIV/INAMI data, low case-volume centers are underrepresented in this survey. The most common ERCPindication was stone extraction $(52 \%) .74 \%$ of endoscopists had more than 10 years of experience in performing ERCP. The majority of centers had their own written protocol $(84 \%)$ for microbiological duodenoscope surveillance. Monitoring of cannulation rate and post-ERCP pancreatitis (PEP) was only performed in a minority of centers $(30 \%)$. The majority of centers $(76 \%)$ provided verbal informed consent relating to the ERCP-procedure ; a minority also requested a written informed consent $(23 \%) .65 \%$ of centers systematically use NSAIDs for PEP-prophylaxis.

Conclusion: This is the first survey of ERCP performance in Belgium. There were wide variations in practice. Adherence to key performance measures and measurement and evaluation of ERCP performance in daily practice at center and endoscopist level are not uniformly widespread. (Acta gastroenterol. belg., 2021, 84, 7377).
\end{abstract}

Keywords : ERCP, quality performance, key performance measures.

\section{Introduction}

Promoting good practice in endoscopy and adherence to specific quality indicators is one of the major priorities of the Belgian Society of Gastrointestinal Endoscopy (BSGIE). In view of the annual BSGIE postgraduate meeting in September 2018, a national survey was performed on the subject of endoscopic retrograde cholangiopancreaticography practice in Belgium. In addition, the statistical department of the National Institute for Health and Disability Insurance (RIZIV/INAMI) provided reimbursement data of all ERCP procedures performed in 2017. The 2018 BSGIE September meeting was fully dedicated to all aspects of ERCP practice and, in particular, to quality in ERCP. ERCP is regarded as one of the most technically challenging endoscopic procedures with relative high complication rates, especially in less experienced hands, although there is some conflicting data on the relationship between endoscopist experience and adverse events (1-7).

Training in ERCP has a long learning curve in both technical endoscopic skills and judgment/interpretation, while ERCP performance progressively is transforming to a platform for more complex advanced diagnostic and therapeutic techniques, such as cholangioscopy and ERCP in altered anatomy.

The purpose of this survey was to assess the current status in ERCP practice, credentialing and quality control in Belgium in order to develop quality improvement strategies. The development of quality registries in certain endoscopic and surgical procedures demonstrates the increased interest and awareness of governmental health care authorities for quality in endoscopy and practice of medicine in general. In view of these quality improvement initiatives, national medical societies should act as an advisory board in defining key performance measures for which they should take joint responsibility.

\section{Materials and methods}

In June 2018, an online questionnaire was sent to all BSGIE-members $(n=116)$. This questionnaire consisted of 32 questions that were reviewed by the BSGIE board members. Only one response per hospital was required (one survey per hospital site in case of a hospital network). Deadline was August, 31th 2018. Answers were anonymized.

The questions were divided into several categories : yearly case volume (2017), baseline endoscopist characteristics and profile (such as lifetime numbers of ERCPs performed and center volume), practice environment of ERCP (room, anaesthesiology), availability for emergencies, prophylaxis of ERCP complications, indications of transfer of patient, quality issues in ERCP (disinfection protocol, informed consent, monitoring of performance).

\footnotetext{
Correspondence to: C. Snauwaert, M.D., Department of Hepatology and Gastroenterology, AZ Sint-Jan Brugge-Oostende AV, Ruddershove 10, 8000 Bruges. Tel. : 0504521 80. Fax : 050452179 E-mail: christophe.snauwaert@azsintjan.be

Submission date : 30/04/2020 Acceptance date : 03/07/2020
} 
Endoscopists who consented to participating in this survey could gain secured access to the web-based questionnaire from June 2018 to August 2018.

Data on total number of ERCPs performed in Belgium were obtained through the statistical department of the RIZIV/INAMI. The total number of ERCPs performed in Belgium could be derived from the total of unique procedural codes that are attributed to the financial reimbursement of ERCP-procedures.

\section{Results}

\section{Total numbers and general data}

In total, 45 endoscopists from 45 different centers (Flanders : 26 ; Wallonia : 14 ; Brussels : 5) completed the online questionnaire. Two responding hospitals did not perform any ERCPs.

Given the data provided by the responders in 2017 , 8368 ERCPs were performed by 113 endoscopists in their centers.

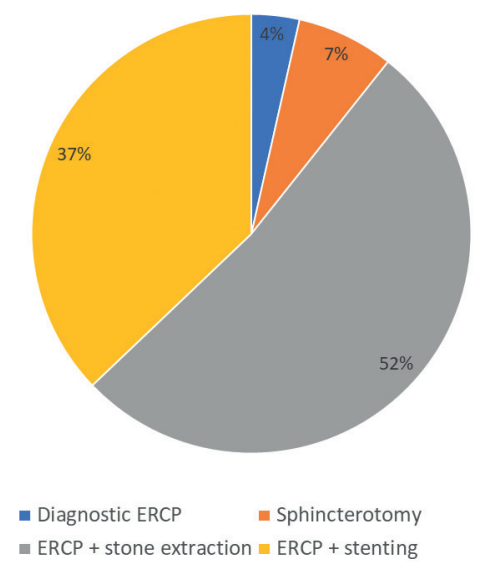

Fig. 1. - Distribution of the different RIZIV/INAMI codes (ERCP indications) that were used annually by the responders.

According to the annual reimbursement RIZIV/ INAMI data for 2017, 12411 ERCPs were performed in 95 centers by 322 endoscopists. This indicates that the current survey might provide a representative picture of the ERCP landscape in Belgium (two thirds of total number of ERCP's annually performed in Belgium).

With regard to the indication for the ERCP examination in general, the RIZIV/INAMI code ERCP + stone extraction was most frequently used (52\%). A circle diagram with the distribution of the different RIZIV/ INAMI codes that were used annually by the responders is displayed in Figure 1.

\section{Center specific data}

$58 \%(25)$ of centers performed $>100$ ERCPs/year and $7 \%$ of centers (3) performed $<50$ ERCPs / year (Figure 2).

According to the annual reimbursement RIZIV/ INAMI data for $2017,51 \%$ of centers (46) performed > 100 ERCPs/year and $26 \%$ of centers (25) performed less than 50 ERCPs/year. This indicates that low case volume centers are underrepresented in this BSGIE survey.

Of the 43 hospitals that performed ERCP and returned the online questionnaire, $21(49 \%)$ provided an ERCP service for their local population only ; $6(14 \%)$ received referrals from other hospitals in their hospital network and $16(37 \%)$ received referrals from hospitals outside their network.

$74 \%$ of centers performing ERCP also refer patients to another facility (mostly tertiary university hospitals) for ERCP-procedures. The rate of referral is rather low with less than 5 patients / year in the majority of centers. Main reasons for possible referral were : failed cannulation (46\%), lithotripsy (50\%), treatment of chronic pancreatitis (34\%), necrosectomy (43\%), cholangioscopy (68\%) and ERCP in altered anatomy (44\%).

\section{Endoscopist characteristics}

The annual ERCP load per endoscopist in relation to the total number of ERCPs per center is demonstrated in Figure 3. In 25 centers (58\%) the annual ERCP load per endoscopist was 50 or higher. $45 \%$ of endoscopists who perform ERCP perform EUS as well.

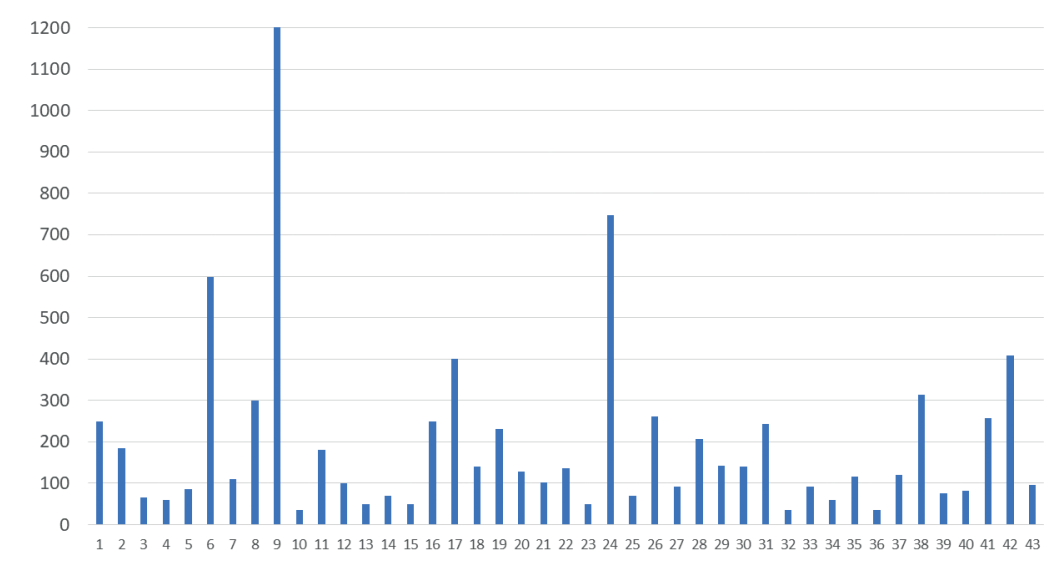

Fig. 2. - Annual ERCPs per center. 


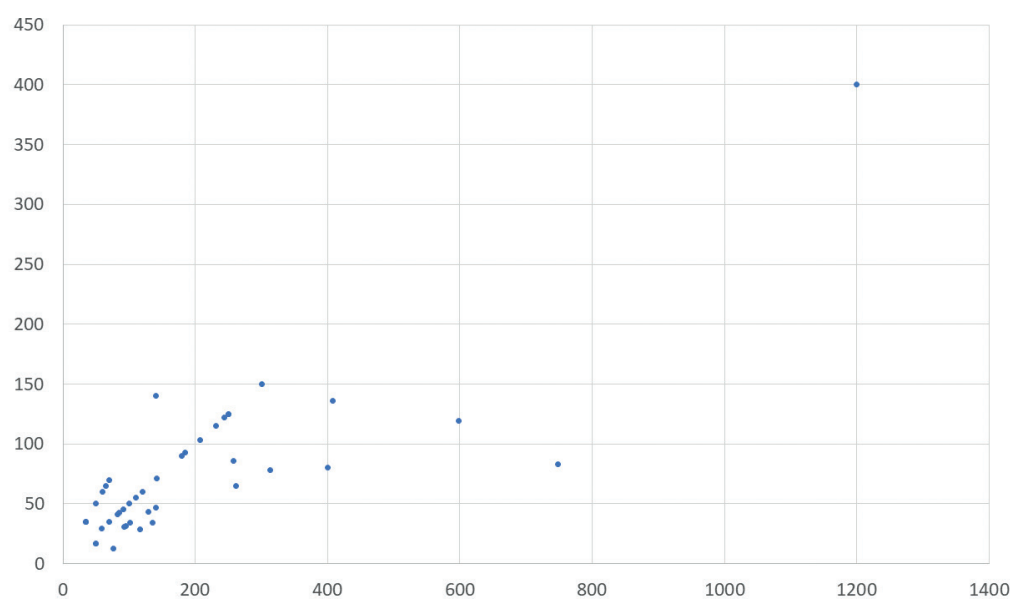

Fig. 3. - Annual ERCPs per endoscopist according to annual ERCPs per hospital.

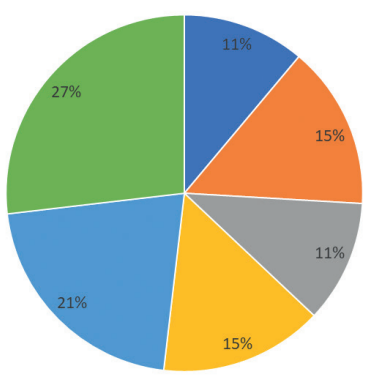

$-<5$ years experience $\quad \mathbf{5}$ to 10 years experience $\| 10$ to 15 years experience ॥ 15 to 20 years experience $\| 20$ to 25 years experience $\|>25$ years experience

Fig. 4. - Endoscopists' years of experience in ERCP.

$74 \%$ of the endoscopists had more than 10 years of experience in performing ERCP (Figure 4). 95\% of responders deemed their case volume of ERCP is sufficient to maintain quality and performance. With reference to the question "do you think some of the endoscopists of your unit should stop performing ERCP", only $5 \%$ of responders answered "yes".

\section{ERCP environment and logistical considerations}

$44 \%$ of centers performed their ERCP-procedures in the radiological department and $28 \%$ in the operating theatre. Only a minority of centers $(28 \%)$ has access to a dedicated fluoroscopic ERCP equipment inside the endoscopy unit.

The majority of centers perform ERCP under general anaesthesia $(88 \%)$ in which patients mostly receive endotracheal intubation (74\%). Urgent ERCP $<24$ h (i.e. in case of cholangitis) can be performed in most centers (84\%), including weekends.

\section{Duodenoscope surveillance}

Concerning the microbiological surveillance of duodenoscopes, the majority of centers had their own written protocol (84\%) for duodenoscope disinfection. $63 \%$ of centers had a systematic duodenoscope microbiological surveillance protocol. $9 \%$ of centers already had experience with multi-drug resistant infections from contaminated duodenoscopes (1 vanco-

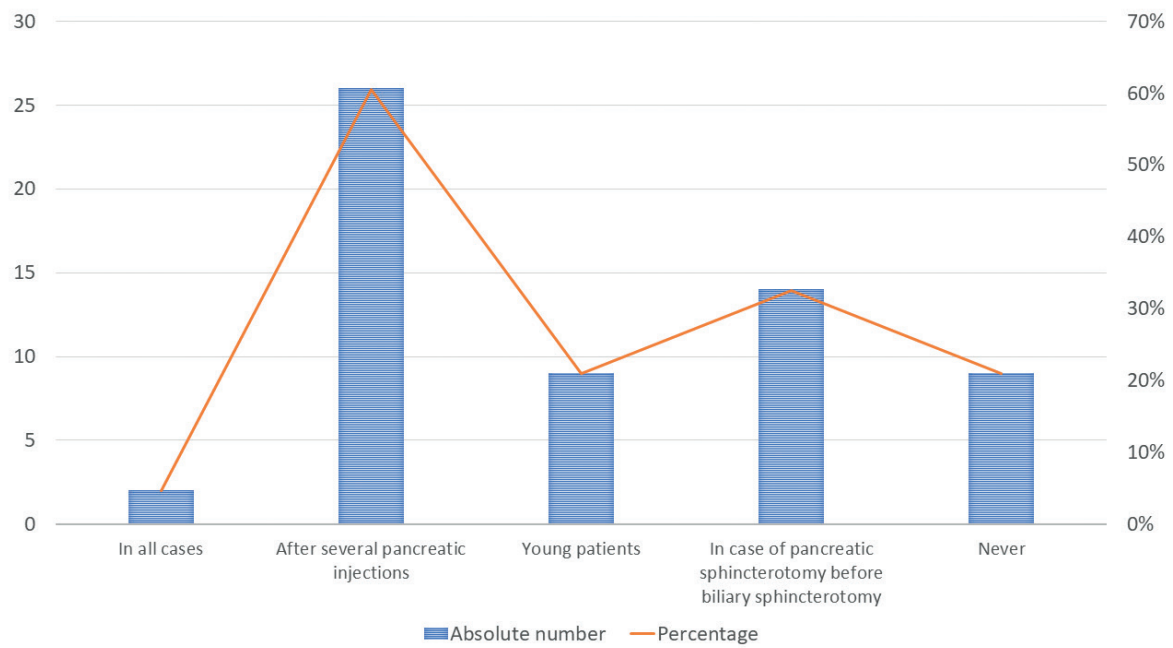

Fig. 5. - Distribution of various situations in which a pancreatic stent is placed. 
mycin resistant Enterococcus (VRE), 3 Extended Spectrum Beta-Lactamase (ESBL) producing bacteria). $66 \%$ of centers store their duodenoscopes in a drying cabinet.

\section{Quality control}

Regarding key performance measures in ERCP, monitoring of cannulation success rate, post-ERCP pancreatitis (PEP) and occurrence of other post-ERCP complications was only performed in a minority of centers $(30 \%)$. Real data regarding procedural outcome of ERCPs are not available since this was not asked to the survey respondents.

In the majority of centers $(76 \%)$ patients provided verbal informed consent relating to the ERCP-procedure (indications, alternative, risks and complications) - a minority of centers also requested a written informed consent $(23 \%)$.

$65 \%$ of centers systematically use NSAIDs prophylaxis in order to prevent PEP while the other centers use NSAIDs in specific situations. Only $2 \%$ of centers never use NSAIDs PEP prophylaxis. 7\% of responders place a prophylactic pancreatic stent in every case of unintended pancreatic cannulation, while two third of responders place a prophylactic stent in case of several unintended pancreatic injections. Distribution of the various situations in which a prophylactic stent is presented in Figure 5.

\section{Discussion}

In this study an online survey was performed regarding the performance of ERCP in Belgium.

According to the real-life RIZIV/INAMI data, in 2017, 12411 ERCPs were performed in 95 centers by 322 endoscopists. In contrast with other therapeutic endoscopic interventions, there is no evidence of increasing demand for ERCP procedures. As a diagnostic test, its role will further decrease (as demonstrated in Figure 1) as access to competing less invasive technologies such as magnetic resonance and endoscopic ultrasound expands.

The current survey provides a representative picture of the ERCP landscape in Belgium (two thirds of ERCPs annually performed in Belgium according to the RIZIV/ INAMI data). However, unfortunately, due to low response rate for the low case-volume centers $(<50$ per year), these are underrepresented in this survey.

The ERCP case volume per hospital but also per endoscopist can raise questions in some centers. For instance, a recent nationwide study performed in the Netherlands demonstrated a significant association between ERCP volume per endoscopist and procedural outcome (7). In this study a yearly volume of $\geq 50$ ERCPs (per endoscopist) was significantly associated with a lower risk of procedural failure, which is in accordance with earlier studies (7-10). This arbitrary threshold of
50 annual ERCPs per endoscopist can only be reached in large-volume centers which is also illustrated by the current survey (Figure 3). On the other hand, the study in the Netherlands also proved that establishing a threshold of 50 procedures per year alone is not enough ; experience seems equally important. Well-experienced endoscopists are sufficiently represented in this Belgian survey with $74 \%$ of responders having more than 10 years of experience in performing ERCP (Figure 4). Moreover, the vast majority of responders judged their colleagues' ERCP-related performance is sufficient to continue to execute ERCPs.

Despite the fact that responders were well-experienced, there was low adherence to specific key performance measures for ERCP (11). For example, only a minority of centers systematically performed monitoring of bile duct cannulation rate and occurrence of post-ERCP pancreatitis.

Furthermore, the application of prophylactic measures recommended by ESGE guidelines in order to prevent post-ERCP pancreatitis is not uniformly prevalent and remains operator dependent (2).

There are increasing concerns about medical and legal issues and the demands for established informed consent guidelines. ESGE guidelines recommend that endoscopists provide written information to individuals undergoing ERCP, and inform patients of therapeutic alternatives and substantial associated risks (defined as pancreatitis, cholangitis, perforation and bleeding).

Nevertheless, our survey demonstrates that standardized practices of patient informed consent for ERCP are not widespread.

Moreover, regarding the bacteriological surveillance of duodenoscopes, about two-thirds of centers use a systematic duodenoscope microbiological surveillance protocol which highlights that also in this specific domain there are opportunities for enhancement. ESGE guidelines recommend 3-monthly microbiological surveillance of a proportion of the department's endoscopes, with the requirement of testing all endoscopes at least once a year (12).

Measurement of procedural outcome is intended to promote the development of quality improvement initiatives (11). There is sufficient evidence showing that the crucial step in measurement of quality necessitates the implementation of systematic recording of key performance measures. The present study demonstrates that measurement of procedural outcome and recording of other key performance measures is currently lacking in Belgium.

Additionally, centralization of complex endoscopical procedures may be another valid strategic approach in order to improve quality performance. Advanced procedures such as ERCP should only be performed in an high-quality and environmentally adapted manner in experienced (clinically and scientifically) institutions in a context of continuous technological development and quality improvement measures (11). Furthermore, 
performing ERCP in dedicated high-volume centers with access to percutaneous transhepatic cholangiography and EUS may also reduce the need for referrals or reinterventions (by combining the procedure during the same anesthesia session) (13-14). Centralization of ERCP procedures in high-volume centers may also improve the pre- and postprocedural management (e.g. administration of NSAIDs, fluid management) of patients by more experienced staff members and nurses. Regionalization or centralization may produce favorable outcome gains. More recently, in the context of complex surgery, conventions have been set up between the RIZIV/INAMI and several recognized medical centers (with sufficient case volume and satisfactory expertise) for the reimbursement of complex esophageal surgery and complex pancreatic surgery. The main objective of this policy is to obtain a high number of complex surgical procedures being performed by more experienced physicians at higher-volume centers, with a concomitant improvement in short-term outcomes. As quality may increase with annual case load, concentration of ERCP procedures in high-volume centers may be beneficial for patient outcomes and may reduce the need for reinterventions and associated costs.

Nevertheless, as recording of key performance measures is not widespread in Belgium, measurement of quality / procedural outcome in ERCP (in low and high volume centers) should be prioritized before focussing on centralization as a quality improvement initiative.

\section{Conclusion}

This online survey provides a valid insight into current ERCP practice in Belgium. However, the low case volume centers (<50 ERCPs/year) are underrepresented in this survey. The annual number of ERCP procedures performed (per endoscopist) is an important determinator of ERCP outcome. The ERCP case volume per hospital but also per endoscopist should raise questions in some centers. The application of prophylactic measures recommended by the ESGE guidelines to prevent post ERCP pancreatitis is not uniformly widespread and remains operator dependent. Quality practices of patient informed consent, bacteriological surveillance of endoscopes and monitoring of performance need improvement. Only a minority of centers systematically monitors ERCP procedural outcome. Institutions offering ERCP to patients should be stimulated to survey quality of their practice by an audit and feedback process in order to identify inadequacies, preserve procedural capacities and enhance clinical practice on a long-term basis.

\section{Conflict of interest statement}

The authors whose names are listed on this title page certify that they have no affiliations with or involvement in any organization or entity with any financial interest (such as honoraria; educational grants; participation in speakers' bureaus ; membership, employment, consultancies), or non-financial interest (such as personal or professional relationships, affiliations, knowledge or beliefs) in the subject matter or materials discussed in this manuscript.

\section{References}

1. FREEMAN ML, NELSON DB, SHERMAN S, HABER GB, HERMAN ME, DORSHER PJ. et al. Complications of Endoscopic Biliary Sphincterotomy. N. Engl. J. Med., 1996, 335(13) : 909-18.

2. DUMONCEAU JM, KAPRAL C, AABAKKEN L, PAPANIKOLAOU IS, TRINGALI A, VANBIERVLIET G. et al. ERCP-related Adverse Events: European Society of Gastrointestinal Endoscopy (ESGE) Guideline. Endoscopy, 2020, 52(2) : 127-149.

3. COTTON PB, GARROW DA, GALLAGHER J, ROMAGNUOLO J. Risk factors for complications after ERCP: a multivariate analysis of 11,497 procedures over 12 years. Gastrointest. Endosc., 2009, 70 : 80-88.

4. COTTON PB, EISEN GM, AABAKKEN L, BARON TH, HUTTER MM, JACOBSON BC. et al. A lexicon for endoscopic adverse events : report of an ASGE workshop. Gastrointest. Endosc., 2010, 71 : 446-454.

5. KESWANI RN, QUMSEYA BJ, O'DWYER LC, WANI S. Associa-tion Between Endoscopist and Center Endoscopic Retrograde Cholangiopancreatography Volume With Procedure Success and Adverse Outcomes: A Systematic Review and Meta-analysis. Clin. Gastroenterol. Hepatol., 2017, 15(12) : 1866-1875

6. LEE HJ, CHO CM, HEO J, JUNG MK, KIM TN, KIM KH. et al. Impact of Hospital Volume and the Experience of Endoscopist on Adverse Events Related to Endoscopic Retrograde Cholangiopancreatography: A Prospective Observational Study. Gut and Liver, 2020, 14(2) : 257-264.

7. EKKELENKAMP VE, DE MAN RA, TER BORG F, BORG PC, BRUNO MJ, GROENEN M.J. et al. Prospective evaluation of ERCP performance: results of a nationwide quality registry. Endoscopy, 2015, 47(6) : 503-7.

8. PENG C, NIETERT PJ, COTTON PB, LACKLAND DT, ROMAGNUOLO J. Predicting native papilla biliary cannulation success using a multinational Endoscopic Retrograde Cholangiopancreatography (ERCP) Quality Network. BMC Gastroenterol., 2013, 13 : 147.

9. KAPRAL C, DULLER C, WEWALKA F, KERSTAN E, VOGEL W, SCHREIBER F. Case volume and outcome of endoscopic retrograde cholangiopancreatography: results of a nationwide Austrian benchmarking project. Endoscopy, 2008, $40: 625-630$.

10. COTE GA, IMLER TD, XU H, TEAL E, FRENCH DD, IMPERIALE TF. et al. Lower provider volume is associated with higher failure rates for endoscopic retrograde cholangiopancreatography. Med. Care, 2013, 51 : 1040-1047.

11. DOMAGK D, OPPONG KW, AABAKKEN L, CZAKÓ L, GYÖKERES T, MANES G. et al. Performance measures for ERCP and endoscopic ultrasound: a European Society of Gastrointestinal Endoscopy (ESGE) Quality Improvement Initiative. Endoscopy, 2018, 50(11) : 1116-1127.

12. BEILENHOFF U, BIERING H, BLUM R, BRLJAK J, CIMBRO M, DUMONCEAU JM et al. Prevention of multidrug-resistant infections from contaminated duodenoscopes: Position Statement of the European Society of Gastrointestinal Endoscopy (ESGE) and European Society of Gastroenterology Nurses and Associates (ESGENA). Endoscopy, 2017, 49(11): 1098-1106.

13. GUO J, GIOVANNINI M, SAHAI AV, SAFTOIU A, DIETRICH CF, SANTO E. et al. A multi-institution consensus on how to perform EUS-guided biliary drainage for malignant biliary obstruction. Endosc. Ultrasound, 2018, 7(6) : 356-365.

14. DHIR V, KHASHAB MA. EUS-guided biliary drainage : Moving beyond the cliché of prime time. Endosc. Ultrasound, 2019, 8 : S1-S2. 